\title{
Promoter hypermethylation and downregulation of the FAS gene may be involved in colorectal carcinogenesis
}

\author{
MEHDI MANOOCHEHRI ${ }^{1,2}$, NASIM BORHANI ${ }^{2}$, ASHRAF KARBASI ${ }^{3}$, \\ AMENEH KOOCHAKI $^{4}$ and BAHRAM KAZEMI ${ }^{1,4}$ \\ ${ }^{1}$ Biotechnology Department, School of Medicine, Shahid Beheshti University of Medical Sciences, \\ Tehran 19857-17453; ${ }^{2}$ School of Medicine, Shahroud University of Medical Sciences, Shahroud 36247-73955; \\ ${ }^{3}$ Gastroenterology and Hepatology Department, Baqiyatallah Research Center for Gastroenterology and Liver Diseases, \\ Baqiyatallah University of Medical Sciences, Tehran 14359-15371; ${ }^{4}$ Cell and Molecular Biology Research Center, \\ Shahid Beheshti University of Medical Sciences, Tehran 19857-17453, Iran
}

Received February 12, 2015; Accepted April 1, 2016

DOI: $10.3892 / \mathrm{ol} .2016 .4578$

\begin{abstract}
Aberrant DNA methylation has been investigated in carcinogenesis and as biomarker for the early detection of colorectal cancer (CRC). The present study aimed to define the methylation status in the regulatory elements of two proapoptotic genes, Fas cell surface death receptor (FAS) and BCL2-associated X protein (BAX). DNA methylation analysis was performed in tumor and adjacent normal tissue using HpaII/MspI restriction digestion and methylation-specific polymerase chain reaction (PCR). The results observed downregulation of the FAS and BAX genes in the CRC tissues compared with the adjacent normal samples. Furthermore, demethylation using 5-aza-2'-deoxycytidine treatment followed by reverse-transcription quantitative PCR were performed on the HT-29 cell line to measure BAX and FAS mRNA expression following demethylation. The 5-aza-2'-deoxycytidine treatment resulted in significant FAS gene upregulation in the HT-29 cell line, but no significant difference in BAX expression. Furthermore, analysis of $\mathrm{CpG}$ islands in the FAS gene promoter revealed that the FAS promoter was significantly hypermethylated in $53.3 \%$ of tumor tissues compared with adjacent normal samples. Taken together, the results indicate that decreased expression of the FAS gene due to hypermethylation of its promoter may lead to apoptotic resistance, and acts as an important step during colorectal carcinogenesis.
\end{abstract}

Correspondence to: Professor Bahram Kazemi, Biotechnology Department, School of Medicine, Shahid Beheshti University of Medical Sciences, Daneshjoo Boulevard, Velenjak, Tehran 19857-17453, Iran

E-mail:kazemi@sbmu.ac.ir

Key words: apoptosis, colorectal cancer, gene expression, Fas cell surface death receptor, methylation

\section{Introduction}

Colorectal cancer (CRC) is the third most common malignancy and third leading causes of cancer-related mortality (9-10\% incidence and mortality rates) in the United States. The risk of developing invasive CRC within a lifetime is estimated to be $\sim 5 \%$ (1). Due to increased early detection and precancerous lesion removal using screening methods in recent years, the rate of CRC-related mortality is being reduced in United States, however, this rate is increasing in developing countries (2). For example, previous studies have shown that the incidence of CRC is increasing in Iran (3-5). Furthermore, due to the high proportion of young people in the Iranian population, the incidence rate of different types of cancer, such as CRC, is expected to increase in the coming years as the population grows older.

It has been suggested that three major pathways are involved in colorectal tumorigenesis, including chromosomal instability, microsatellite instability and the $\mathrm{CpG}$ island methylator phenotype (CIPM) (6). Previous studies have demonstrated that epigenetic modifications, such as aberrant DNA methylation, have important roles during CRC development. CIPM-positive CRC cases, which account for $\sim 15 \%$ of sporadic cases, have hypermethylation in the promoter regions of certain tumor suppressor genes $(7,8)$. CpG islands are genomic regions with a high percentage of $\mathrm{CpG}$ dinucleotides. DNA methylation occurs at $\mathrm{CpG}$ dinucleotides, and has been demonstrated to suppress the expression of nearby genes (9). There are $25,500 \mathrm{CpG}$ islands in the human genome, half of which are associated with constitutively expressed genes; a number of these constitutively expressed genes are methylated in CRC (10). In a study by Kim et al, the previously reported methylation markers has been reviewed in CRC (11). Epigenetic modifications, such as promoter hypermethylation, commonly precede disease pathology, making them valuable diagnostic biomarkers for the early detection of diseases or for determining clinical response to the therapeutics $(12,13)$. Furthermore, DNA methylation markers are more stable compared with RNA and protein markers, thus, making them more suitable biomarkers for detection in different biological substances, such as blood and stool samples (14). 
Apoptosis or programmed cell death is an important homeostatic process; its failure is considered to contribute to cancer development and resistance to cancer therapies (15). Apoptosis occurs through two interconnected pathways; the extrinsic and the intrinsic pathways. The extrinsic pathway initiates by the binding of death ligands to their respective cell surface death receptors: Tumor necrosis factor receptor 1, Fas cell surface death receptor (Fas), and tumor necrosis factor receptor superfamily members $10 \mathrm{a}$ and $10 \mathrm{~b}$. Ligand binding leads to caspase activation and subsequent apoptotic signaling cascades (16). By contrast, the intrinsic or mitochondrial pathway is triggered by oxidative stress and DNA damage, and progresses through proteolytic caspase cascades. The key elements of apoptosis include pro-apoptotic and anti-apoptotic proteins that are involved in the progression and regulation of apoptosis $(17,18)$. Molecular alterations, such as genetic mutations or epigenetic silencing of apoptotic genes, frequently occur in tumor progression and drug resistance (19). As an epigenetic mechanism of gene inactivation, aberrant DNA methylation, in addition to genetic mutations, may act as a second hit to turn off important tumor suppressor genes, and initiate or progress cancer development (20).

Proapoptotic genes that have important roles in apoptosis initiation and progression may contain $\mathrm{CpG}$ islands in their promoter sequences; hence, these genes are prone to be silenced by DNA hypermethylation. In our previous study, two proapoptotic genes, FAS and BCL2-associated $\mathrm{X}$ protein (BAX), were demonstrated to be downregulated in adjacent normal colorectal tissue compared with CRC tissue samples (21). As a cell surface death receptor, Fas protein leads to the initiation of programmed cell death following binding to its ligand. By contrast, Bax is considered to have a significant effect on mitochondrial outer membrane permeabilization; this permeabilization allows cytochrome $c$ to be directed from the mitochondrial intermembrane space into the cytoplasm, and eventually leads to activation of caspases during apoptosis initiation and progression (22). Both the FAS and BAX genes have $\mathrm{CpG}$ islands in their upstream sequences according to the MethPrimer criteria (23). The cell death receptor FAS is involved in initiating the extrinsic pathway of apoptosis, while the BAX protein has important roles in the intrinsic and extrinsic apoptotic pathways (24). Therefore, the aim of the present study was to investigate the methylation status of $\mathrm{CpG}$ islands in two essential downregulated proapoptotic genes, FAS and BAX, in CRC samples.

\section{Materials and methods}

Patient samples. Fresh-frozen patient samples obtained from hospitals were provided by the Iran National Tumor Bank (Tehran, Iran), which is funded by the Cancer Institute of Tehran University (Tehran, Iran; Table I). A total of 30 colorectal tumor samples and their adjacent normal tissues were collected from Baqiyatallah Hospital (Tehran, Iran) and Imam Khomeini Hospital Complex (Tehran, Iran). Samples were collected during surgical resection between March 2011 and September 2012. The criteria for inclusion of patient samples was the sporadic colon cancer, and rectum mucinous and non-mucinous adenocarcinoma. Tumors were classified based on the pathological diagnostic criteria of the WHO classification (25). No patients underwent chemotherapy prior to surgery and had no other forms of cancer. The adjacent normal tissues were obtained from at least $6 \mathrm{~cm}$ away from the tumor sites. The samples were snap-frozen and stored in liquid nitrogen until extraction. The study was approved by Shahid Beheshti University of Medical Sciences ethical committee (Tehran, Iran) and written informed consent was obtained from all patients or close relatives for sampling.

Cell culture and treatment. The HT-29 colorectal adenocarcinoma cell line (ATCC HTB-38; American Type Culture Collection, Manassas, VA, USA) was cultured in RPMI-1640 medium (Biosera, Sussex, UK) supplemented with $10 \%$ heat-inactivated fetal bovine serum and $1 \%$ penicillin-streptomycin (Life Technologies; Thermo Fisher Scientific, Inc., Waltham, MA, USA) at $37^{\circ} \mathrm{C}$ in a humidified atmosphere with $5 \% \mathrm{CO}_{2}$. The cells were cultured in 6-well plate at equal concentrations of $\sim 1 \times 10^{4}$ cells per well. Subsequently, the cells were exposed media containing $10 \mu \mathrm{M}$ 5-aza-2'-deoxycytidine (Sigma-Aldrich Chemie GmbH, Hamburg, Germany) for $48 \mathrm{~h}$ to induce DNA demethylation, while control cells were left untreated. After $24 \mathrm{~h}$, the media was changed and replaced with fresh media containing $10 \mu \mathrm{M}$ 5-aza-2'-deoxycytidine.

RNA extraction and reverse transcription-quantitative polymerase chain reaction ( $R T-q P C R)$. To minimize gene expression variations, lysis buffer was poured directly on the cells in the wells. Total RNA from the 5-aza-2'-deoxycytidine-treated and untreated control cells was extracted after $48 \mathrm{~h}$ exposure using an AllPrep DNA/RNA Mini kit (Qiagen, Valencia, CA, USA). cDNA was synthesized using a PrimeScript RT Reagent kit (Takara Bio, Inc.), and the expression of the FAS and BAX genes were measured in the 5-aza-2'-deoxycytidine-treated and untreated HT-29 cells. qPCR was performed under the following conditions: $30 \mathrm{sec}$ initial denaturation step at $95^{\circ} \mathrm{C}$ followed by 40 amplification cycles at $94^{\circ} \mathrm{C}$ for $5 \mathrm{sec}$ and $60^{\circ} \mathrm{C}$ for $30 \mathrm{sec}$. Melting curve analysis was then performed. Relative quantification of the FAS and BAX genes was performed by RT-qPCR in a Rotor-Gene 6000 cycler (Corbett Life Science, Sydney, Australia) using SYBR Premix Ex Taq II (Takara Bio, Inc.), and the GAPDH gene was used as a positive internal control, whilst no template control NTC reactions served as negative controls for nucleic acid contamination and primer dimer formation. The primer sequences for RT-qPCR were extracted from PrimerBank (http://pga.mgh.harvard.edu/primerbank/) (Table II). RT-qPCR was repeated twice for relative quantification analysis, which was performed using the $\Delta \Delta \mathrm{Cq}$ method (26).

Methylation-sensitive restriction enzyme PCR. DNA and RNA were extracted simultaneously from tumoral and normal adjacent tissues using an AllPrep DNA/RNA Mini kit. The extracted DNA samples were digested using methylation-sensitive HpaII and methylation-insensitive $M s p$ I restriction enzymes (Fermentas EpiJET DNA Methylation Analysis kit; Thermo Fisher Scientific, Inc.). The primers for the PCR reactions were designed using Primer3 software v0.4.0 (http://frodo.wi.mit.edu/primer3), wherein the primers flank the HpaII/MspI enzyme cutting site 
Table I. Clinical data of 25 colorectal cancer samples.

\begin{tabular}{lc}
\hline Characteristic & Cases, $\mathrm{n}(\%)$ \\
\hline Gender & \\
Male & $14(56)$ \\
Female & $11(44)$ \\
Age, years & \\
$<60$ & $15(60)$ \\
$\geq 60$ & $10(40)$ \\
Site of primary tumor & \\
Colon & $13(52)$ \\
Rectum & $12(48)$ \\
Histological type & \\
Non-mucinous adenocarcinoma & $20(80)$ \\
Mucinous adenocarcinoma & $5(5)$ \\
Tumor grade & \\
I & $15(60)$ \\
II & $5(20)$ \\
III & $4(16)$ \\
IV & $1(4)$ \\
T classification & \\
T2 & $4(16)$ \\
T3 & $21(84)$ \\
\hline
\end{tabular}

Table II. Reverse transcription-quantitative polymerase chain reaction primer sets, extracted from the PrimerBank database.

\begin{tabular}{ll}
\hline Gene name & \multicolumn{1}{c}{ Primer sequence, $5^{\prime} \rightarrow 3^{\prime}$} \\
\hline GAPDH & F: AAGGTGAAGGTCGGAGTCAAC \\
& R: GGGGTCATTGATGGCAACAATA \\
FAS & F: CACCCGGACCCAGAATACC \\
& R: TGTTGCTGGTGAGTGTGCATT \\
BAX & F: CCCGAGAGGTCTTTTTCCGAG \\
& R: CCAGCCCATGATGGTTCTGAT \\
\hline
\end{tabular}

F, forward; R, reverse; GAPDH, glyceraldehyde 3-phosphate dehydrogenase; FAS, Fas cell surface death receptor; BAX, BCL2-associated $\mathrm{X}$ protein.

(5'-CCGG-3') in the sequences. The primers used for amplification were as follows: Forward, 5'-ACTTCCTGCCTCTGG CACT-3' and reverse, 5'-AGGCTGGGCCTGTATCCTAC-3' for BAX gene (GenBank: AF339054.1); forward, 5'-ACGAAC CCTGACTCCTTCCT-3' and reverse, 5'-TCAGAGACGAGC TCACGAAA-3' for FAS gene (GenBank: X82279.1). For PCR amplification, a $25-\mu 1$ reaction volume, including $12.5 \mu 1 \mathrm{Taq}$ DNA Polymerase Master Mix Red from Ampliqon (Odense M,

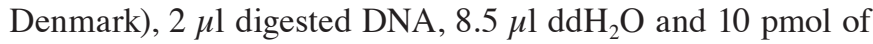
each primer, was added to 0.2-ml Eppendorf microtubes. The reactions were performed for 30 cycles on a Mastercycler ${ }^{\circledR}$ Nexus (Eppendorf, Hamburg, Germany); $95^{\circ} \mathrm{C}$ predenaturation for 4 min followed by denaturation at $94^{\circ} \mathrm{C}$ for $35 \mathrm{sec}$, annealing at $64^{\circ} \mathrm{C}$ for $30 \mathrm{sec}$, extension at $72^{\circ} \mathrm{C}$ for $30 \mathrm{sec}$ and final extension at $72^{\circ} \mathrm{C}$ for $5 \mathrm{~min}$. The amplified products were visualized on $1.5 \%$ agarose gel electrophoresis and gel band pixel quantification was performed using ImageJ software v1.47p (National Institutes of Health, Bethesda, MD, USA).

Bisulfite conversion and methylation-specific PCR (MSP). Sodium bisulfite conversion of genomic DNA was performed on $1 \mu \mathrm{g}$ DNA using an EpiTect Bisulfite kit, according to the manufacturer's protocol (Qiagen, Hilden, Germany). Human control DNA, containing bisulfite-converted methylated and unmethylated DNA (EpiTect PCR Control DNA Set; Qiagen), was used as the control for MSP reactions. The specific primer sets for PCR were designed by MethylPrimer software v1.0 (Thermo Fisher Scientific, Inc.) and/or extracted from studies previously published in the literature $(25,27)$. The primers were as follows: Forward, 5'-AGTGATATATAGGTGTTT AAAGACGT-3' and reverse, 5'-AACTAACCTCAAAATATA TTCCGTA-3' for methylated sequences; forward, 5'-AGT GATATATAGGTGTTTAAAGATGT-3' and reverse, 5'-AAC TAACCTCAAAATATATTCCATA-3' for unmethylated sequences. The PCR protocol was the same as performed for methylation-sensitive restriction enzyme PCR.

Statistical analysis. GraphPad Prism 5 software (GraphPad Software, Inc., San Diego, CA, USA) was used to perform statistical analyses. Student's t-test was applied to compare mean gene expression value between tumor samples and adjacent normal samples. The experiments were repeated twice or in cases of discrepancy they were repeated three times, and all data are expressed as mean \pm standard deviation. $\mathrm{P}<0.05$ was considered to indicate a statistically significant difference.

\section{Results}

$R T$ - $q P C R$. Using RT-qPCR, BAX and FAS gene expression were analyzed in CRC and normal samples according to their pathological grade (I-IV). RT-qPCR revealed downregulation of the two genes (19 out of 30 FAS samples and 18 out of 30 BAX samples) in CRC tissues compared with adjacent normal samples in almost all pathological grades; however, this decrease in expression was more significant in tumor grades I and III (Fig. 1). Furthermore, RT-qPCR demonstrated that FAS gene expression was significantly upregulated in 5-aza-2'-deoxycytidine-treated HT-29 cells compared with untreated HT-29 control cells ( $\mathrm{P}=0.00518)$; however, the difference in BAX gene expression was not significant between treated and untreated control cells (Fig. 2).

Methylation-sensitive restriction enzyme PCR. PCR amplification of the selected region (-166 to -6) was performed in the upstream region of the FAS gene to yield a 160-bp product containing two HpaII/MspI cutting sites. Semi-quantification of agarose gel bands using ImageJ software revealed significant methylation of the desired region of the FAS gene in 19 out of 30 tumor samples compared with adjacent normal samples (Fig. 3).

$M S P$. Two different $\mathrm{CpG}$ rich sections in the regulatory regions of the FAS gene were analyzed by MSP (Fig. 4A). MSP analysis 

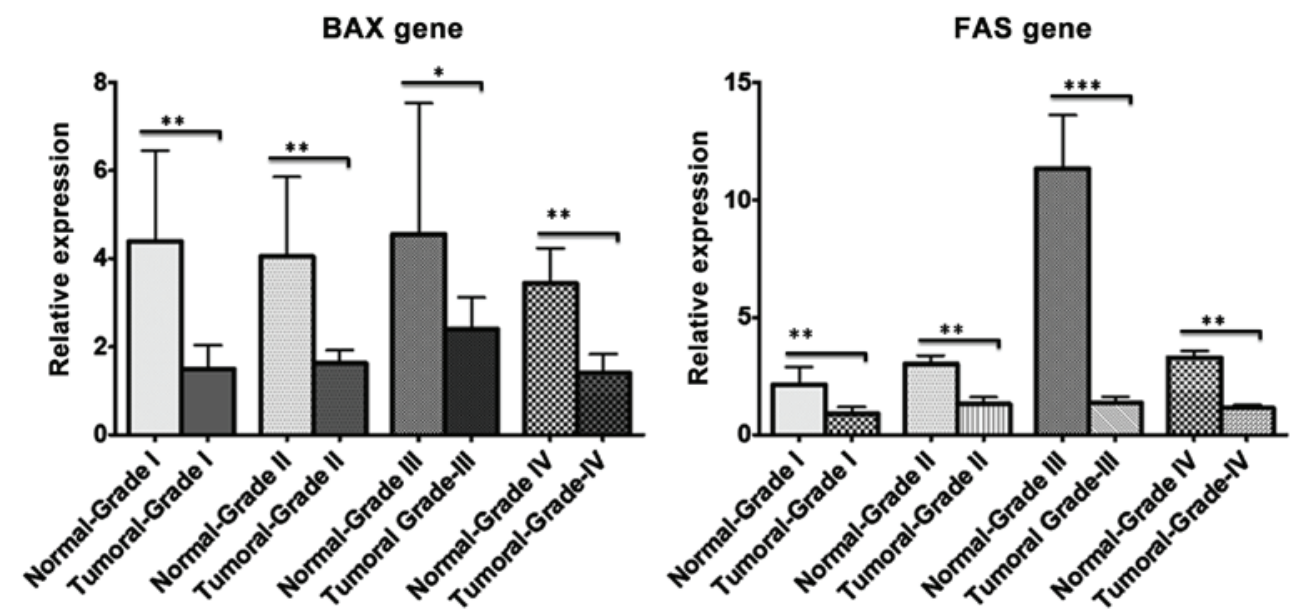

Figure 1. Relative expression of BAX and FAS genes between different grades of colorectal cancer. Stratifying and comparing the reverse transcription-quantitative polymerase chain reaction data from colorectal cancer tumoral and adjacent normal samples between four different grades (I-IV) revealed that BAX and FAS proapoptotic genes were significantly downregulated in tumoral compared with normal samples. $\mathrm{P} \leq 0.05,{ }^{* *} \mathrm{P} \leq 0.01,{ }^{* * * *} \mathrm{P} \leq 0.001$. Graphs were generated using GraphPad Prism software and data are expressed as mean \pm standard deviation. BAX, BCL2-associated X protein; FAS, Fas cell surface death receptor.

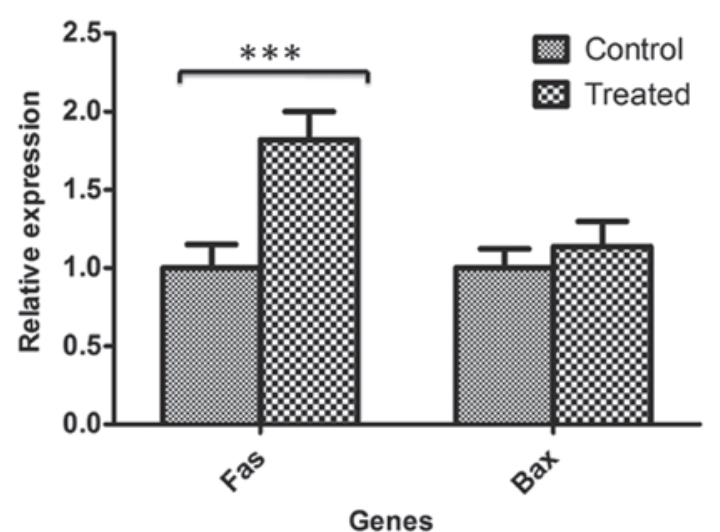

Figure 2. FAS and BAX gene expression variations between 5-aza-2'-deoxycytidine-treated and untreated control HT-29 cells. Reverse transcription-quantitative polymerase chain reaction data revealed that FAS gene was significantly upregulated in 5-aza-2'-deoxycytidine treated cells campared with untreated normal control cells; however, BAX gene expression was not significantly different between two groups. ${ }^{* * *} \mathrm{P} \leq 0.001$. Data are expressed as mean \pm standard deviation. FAS, Fas cell surface death receptor; BAX, BCL2-associated X protein.

of the promoter region revealed marked methylation in the tumor samples compared with the normal adjacent samples (Fig. 4B). The results identified that 16/30 (53.3\%) tumoral samples were markedly methylated in the FAS promoter region compared with the normal samples. To determine if the hypermethylation in the FAS promoter is correlated with its downregulation, the expression of FAS in these 16 hypermethylated samples were compared and 12/16 samples showed significantly lower expression in tumor samples than in normal adjacent samples. The first intron of the FAS gene has a putative enhancer containing a P53 response element. To determine the methylation in the enhancer region of the FAS gene in CRC tissue samples, the MSP technique was performed with sodium bisulfate-treated DNA from tumor and normal adjacent tissues. Methylation status analysis of the FAS enhancer region using MSP indicated no methylation in this region in CRC and normal samples (Fig. 4C).

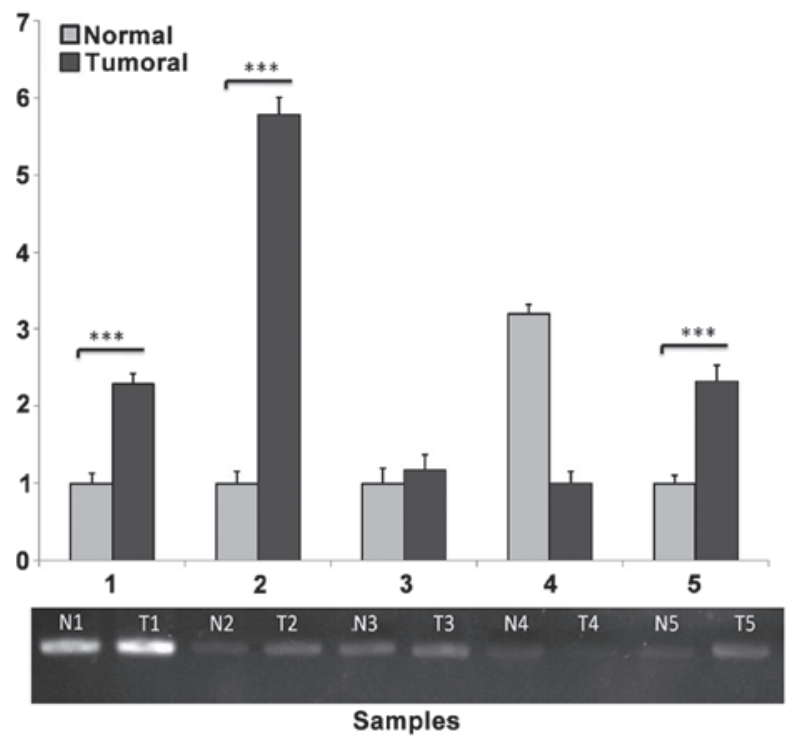

Figure 3. Semi-quantitative methylation-sensitive HpaII-restriction enzyme polymerase chain reaction (PCR) for the FAS gene. PCR following digestion with HpaII demonstrated a significant difference in FAS gene expression between tumor (T) and normal adjacent (N) DNA. Relative pixel digital quantification was obtained using ImageJ software. The tumoral samples shows higher pixel intensity compared with normal samples. ${ }^{* * *} \mathrm{P} \leq 0.001$.

\section{Discussion}

Numerous efforts have been made to identify epigenetic biomarkers that can be used for CRC early detection, progression, tendency to metastasis, prognosis and response to chemotherapeutics (28-30). DNA methylation has been investigated for application in cancer screening of high-risk populations due to its stability compared with protein and RNA biomarkers, and its easy detection using PCR-based approaches. Methylation marks that are methylated more frequently in tumoral samples compared with normal samples may have potential as methylation biomarkers (31). A previous study revealed that the number of neoplasms containing methylated genes is not significantly different between advanced 
a FAS gene GpG islands

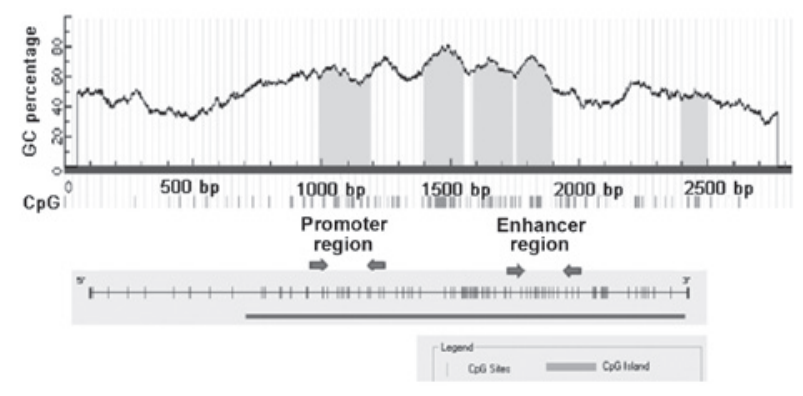

b Promoter

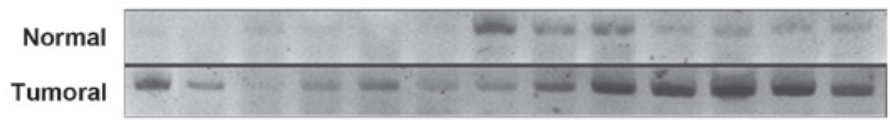

C Enhancer

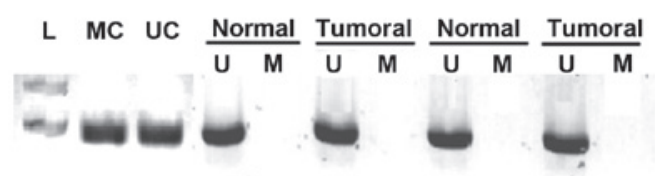

Figure 4. Methylation-specific polymerase chain reaction (MSP) results for FAS promoter and enhancer regions. (A) Analysis revealed that FAS gene has $\mathrm{CpG}$ islands flanking its regulatory sequences. (B) MSP of the promoter region using a methylated specific primer set revealed a higher level of methylation in tumoral samples relative to the normal samples. (C) MSP of enhancer region using methylated and unmethylated specific primer sets on tumoral and normal samples identified no methylation in this region. In the MSP experiments, MC and UC were used to confirm the polymerase chain reaction results. FAS, Fas cell surface death receptor; MC, methylated control; UC, unmethylated control.

colorectal adenoma and colorectal adenocarcinoma; however, these methylated genes are highly different between early colorectal adenoma and colorectal adenocarcinoma (32). Therefore, the methylation of specific gene promoters appears to occur during the early stages of CRC tumorigenesis, and these DNA marks may be classified as useful non-invasive early detection biomarkers if they can be easily detected in available body specimens, such as blood and stool.

In the present study, we hypothesized that DNA methylation of proapoptotic genes that are considered to be tumor suppressor genes may lead to resistance to apoptosis and the development of CRC. Thus, two proapoptotic genes with $\mathrm{CpG}$ islands in their promoter regions and flanking their transcription initiation sites were selected for analysis in the present study. Gene expression evaluation of tumoral and normal adjacent samples demonstrated that the two genes, FAS and BAX, exhibited significantly lower expression in tumoral compared with normal samples in the majority of specimens (Fig. 1). These genes contain $\mathrm{CpG}$ islands flanking the initiation site; hence, methylation and resultant transcriptional silencing may occur in carcinogenesis. Furthermore, methylation inhibition of the HT-29 CRC cell line using 5-aza-2'-deoxycytidine resulted in overexpression of the FAS gene due to DNA demethylation (Fig. 2).

To analyze the $\mathrm{CpG}$ island methylation status, the present study first performed methylation-sensitive restriction enzyme PCR using HpaII and MspI enzymes. In the HpaII assays, no significant difference in methylation status was detected for the BAX gene (data not shown); however, a significant difference in the methylation status of the FAS gene promoter was identified between tumoral and normal specimens in the majority of current samples. To confirm the methylation of $\mathrm{CpG}$ island regions in the FAS gene, the MSP technique was also performed. Two hotspot CG-rich regions in intron 1 and the promoter of the FAS gene were examined for methylation marks. As well as $\mathrm{CpG}$ islands, intron 1 of the FAS gene contains a P53-response element, and is, therefore, considered to be an enhancer region (27).

The MSP results indicated no methylation in the tumoral or normal samples in the enhancer region of the FAS gene. Therefore, methylation of the enhancer region in intron 1 of the FAS gene does not appear to be responsible for FAS downregulation and, thus, based on the current study, is not a causative risk factor for colorectal carcinogenesis (Fig. 4C). This result is in contradiction with a previous study, which identified partial methylation in 4/10 colon carcinoma tumor samples (27). However, the FAS gene has a CpG island around its 5'-flanking region; therefore, DNA methylation in this region may be responsible for the decreased expression of the FAS gene observed in the present tumoral samples. In the present study, MSP was performed using a methylation-specific primer set to confirm the methylation status of the $\mathrm{CpG}$ dinucleotides in the promoter region; the results revealed that the majority of tumoral samples $(53.3 \%)$ contained a markedly higher proportion of methylated FAS compared with the normal samples (Fig. 4B). To determine if the hypermethylation in the FAS promoter is correlated with its downregulation, the expression of FAS in these 16 hypermethylated samples were compared and 12/16 samples showed significantly lower expression in tumor samples than in normal samples $(\mathrm{P}=0.0219)$. Therefore, we propose that methylation in the promoter region may be responsible for downregulating FAS gene expression during CRC carcinogenesis. The current findings are in accordance with a previous study, which demonstrated that FAS promoter methylation is associated with the levels of FAS expression in different CRC cell lines; increased methylation of CpG dinucleotides was associated with lower FAS gene expression (27). This GC-rich promoter region contains binding sites for specificity 
protein-1, activator protein (AP)-1, AP-2, GAGA factor, nuclear factor of activated T-cells and nuclear factor $-\kappa \mathrm{B}$ transcription factors; and methylation of $\mathrm{CpG}$ dinucleotides in these binding sites may affect transcription of the FAS gene (33). By contrast, in another previous study, southern blotting was performed using methylation-sensitive restriction enzymes for FAS promoter methylation evaluation, and revealed no correlation between promoter hypermethylation and loss of FAS expression during colorectal carcinogenesis (34).

In conclusion, the present results demonstrated that FAS promoter hypermethylation is associated with FAS downregulation in CRC but not normal adjacent samples in the investigated population. Furthermore, the FAS promoter exhibited higher levels of methylation in tumoral tissues compared with the normal adjacent tissues. Therefore, FAS promoter methylation levels may be used as an early detection biomarker for patients with CRC; however, confirmatory studies using larger sample sizes non-invasive body specimens, such as blood and/or stool, are required.

\section{Acknowledgements}

We thank Iran National Tumor Bank personnel for their cooperation in providing biological materials. The present study was supported by a grant from Shahid Beheshti University of Medical Sciences (grant no. 9729; Tehran, Iran) and Baqyiatallah University of Medical Sciences (grant no. 1390.22; Tehran, Iran).

\section{References}

1. Jemal A, Siegel R, Xu J and Ward E: Cancer statistics, 2010. CA Cancer J Clin 60: 277-300, 2010.

2. Jemal A, Bray F, Center MM, Ferlay J, Ward E and Forman D: Global cancer statistics. CA Cancer J Clin 61: 69-90, 2011.

3. Mousavi SM, Gouya MM, Ramazani R, Davanlou M, Hajsadeghi N and Seddighi Z: Cancer incidence and mortality in Iran. Ann Oncol 20: 556-563, 2009.

4. Safaee A, Fatemi SR, Ashtari S, Vahedi M, Moghimi-Dehkordi B and Zali MR: Four years incidence rate of colorectal cancer in Iran: a survey of national cancer registry data - implications for screening. Asian Pac J Cancer Prev 13: 2695-2698, 2012.

5. Dolatkhah R, Somi MH, Kermani IA, Ghojazadeh M, Jafarabadi MA, Farassati F and Dastgiri S: Increased colorectal cancer incidence in Iran: A systematic review and meta-analysis. BMC Public Health15: 997, 2015.

6. Worthley DL and Leggett BA: Colorectal cancer: Molecular features and clinical opportunities. Clin Biochem Rev 31: 31-38, 2010.

7. Takayama T, Miyanishi K, Hayashi T, Sato Y and Niitsu Y: Colorectal cancer: Genetics of development and metastasis. J Gastroenterol 41: 185-192, 2006.

8. Issa JP: Colon cancer: It's CIN or CIMP. Clin Cancer Res 14: 5939-5940, 2008.

9. Deaton $\mathrm{AM}$ and Bird $\mathrm{A}: \mathrm{CpG}$ islands and the regulation of transcription. Genes Dev 25: 1010-1022, 2011.
10. Illingworth RS, Gruenewald-Schneider U, Webb S, Kerr AR, James KD, Turner DJ, Smith C, Harrison DJ, Andrews R and Bird AP: Orphan $\mathrm{CpG}$ islands identify numerous conserved promoters in the mammalian genome. PLoS Genet 6: e1001134, 2010.

11. Kim MS, Lee J and Sidransky D: DNA methylation markers in colorectal cancer. Cancer Metastasis Rev 29: 181-206, 2010.

12. Kelly TK, De Carvalho DD and Jones PA: Epigenetic modifications as therapeutic targets. Nat Biotechnol 28: 1069-1078, 2010.

13. Portela A and Esteller M: Epigenetic modifications and human disease. Nat Biotechnol 28: 1057-1068, 2010.

14. Fleischhacker M and Schmidt B: Circulating nucleic acids (CNAs) and cancer-a survey. Biochim Biophys Acta 1775: 181-232, 2007.

15. Iannolo G, Conticello C, Memeo L and De Maria R: Apoptosis in normal and cancer stem cells. Crit Rev Oncol Hematol 66: 42-51, 2008.

16. Oliver L and Vallette FM: The role of caspases in cell death and differentiation. Drug Resist Updat 8: 163-170, 2005.

17. Qiao L and Wong BC: Targeting apoptosis as an approach for gastrointestinal cancer therapy. Drug Resist Updat 12: 55-64, 2009.

18. Ghobrial IM, Witzig TE and Adjei AA: Targeting apoptosis pathways in cancer therapy. CA Cancer J Clin 55: 178-194, 2005.

19. Hector S and Prehn JH: Apoptosis signaling proteins as prognostic biomarkers in colorectal cancer: A review. Biochim Biophys Acta 1795: 117-129, 2009.

20. Jones PA and Baylin SB: The fundamental role of epigenetic events in cancer. Nat Rev Genet 3: 415-428, 2002.

21. Manoochehri M, Karbasi A, Bandehpour M and Kazemi B: Down-regulation of BAX gene during carcinogenesis and acquisition of resistance to 5-FU in colorectal cancer. Pathol Oncol Res 20: 301-307, 2014

22. Rupnarain C, Dlamini Z, Naicker S and Bhoola K: Colon cancer: genomics and apoptotic events. Biol Chem 385: 449-464, 2004.

23. Li LC and Dahiya R: MethPrimer: Designing primers for methylation PCRs. Bioinformatics 18: 1427-1431, 2002.

24. Elmore S: Apoptosis: A review of programmed cell death Toxicol Pathol: 35: 495-516, 2007.

25. Aaltonen LA and Hamilton SR (eds): World Health Organization classification of tumours: Pathology and genetics of tumours of the digestive system. IARC Press, Lyon, 2000.

26. Livak KJ and Schmittgen TD: Analysis of relative gene expression data using real-time quantitative PCR and the 2(-Delta Delta C(T)) Method. Methods 25: 402-408, 2001.

27. Petak I, Danam RP, Tillman DM, Vernes R, Howell SR, Berczi L, Kopper L, Brent TP and Houghton JA: Hypermethylation of the gene promoter and enhancer region can regulate Fas expression and sensitivity in colon carcinoma. Cell Death Differ 10: 211-217, 2003.

28. Coppedè F: Epigenetic biomarkers of colorectal cancer: Focus on DNA methylation. Cancer Lett 342: 238-247, 2014.

29. Choong MK and Tsafnat G: Genetic and epigenetic biomarkers of colorectal cancer. Clin Gastroenterol Hepatol 10: 9-15, 2012.

30. Binefa G, Rodríguez-Moranta F, Teule A and Medina-Hayas M: Colorectal cancer: From prevention to personalized medicine. World J Gastroenterol 20: 6786-6808, 2014.

31. Shivapurkar N and Gazdar AF: DNA methylation based biomarkers in non-invasive cancer screening. Curr Mol Med 10: 123-132, 2010.

32. Lao VV and Grady WM: Epigenetics and colorectal cancer. Nat Rev Gastroenterol Hepatol 8: 686-700, 2011.

33. Singh R, Pradhan V, Patwardhan M and Ghosh K: APO-1/Fas gene: Structural and functional characteristics in systemic lupus erythematosus and other autoimmune diseases. Indian J Hum Genet 15: 98-102, 2009.

34. Butler LM, Dobrovic A, Bianco T and Cowled PA: Promoter region methylation does not account for the frequent loss of expression of the Fas gene in colorectal carcinoma. Br J Cancer 82: 131-135, 2000. 